\title{
MECHANISMS OF AMINO ACID FORMATION IN INTERSTELLAR ICE ANALOGS
}

\author{
Jamie E. Elsila, ${ }^{1,2}$ Jason P. Dworkin, $^{3}$ Max P. Bernstein, ${ }^{1}$ Mildred P. Martin, ${ }^{3,4}$ and Scott A. Sandford ${ }^{1}$ \\ Received 2006 November 20; accepted 2007 January 24
}

\begin{abstract}
Amino acids have been identified in carbonaceous chondrites, but their origin is yet unknown. Previous work has shown that a variety of amino acids can be formed via ultraviolet photolysis of interstellar ice analogs. Two possible mechanisms of formation of these amino acids have been proposed: a Strecker-type synthesis or a radical-radical mechanism. In this work, we have used isotopic labeling techniques to test the predictions made by each of these proposed mechanisms for the formation of the amino acids glycine and serine. We observe that amino acid formation occurs via multiple pathways, with potentially different mechanisms for glycine and serine. The major reaction paths do not match either of the two predicted mechanisms, although a modified radical-radical mechanism may account for our observations. The observation of multiple routes suggests that the formation of amino acids in interstellar ice analogs is not narrowly dependent on ice composition, but may occur under a variety of conditions that influence product distributions.
\end{abstract}

Subject headings: astrobiology — astrochemistry — ISM: molecules — molecular processes

\section{INTRODUCTION}

The understanding of the origin and distribution of extraterrestrial amino acids has been the focus of numerous research studies. Amino acids have been observed in a number of carbonaceous chondrite meteorites of different classes (Kvenvolden et al.1970; Cronin \& Moore 1971; Cronin \& Pizzarello 1983; Pizzarello et al. 1991; Ehrenfreund et al. 2001b; Glavin et al. 2006). Over 80 distinct amino acids have been identified in the Murchison meteorite alone; the indigenous nature of these amino acids is confirmed by the presence of several compounds that are rare or nonexistent in the terrestrial biosphere, by the racemic distributions observed, and by the presence of nonterrestrial isotopic ratios (Oro et al. 1971; Cronin \& Pizzarello 1983; Yuen et al. 1984; Epstein et al. 1987; Pizzarello et al. 1991, 1994; Cronin \& Chang 1993).

The location and mechanism of formation of these meteoritic amino acids remains unknown. Isotopic measurements of amino acids in Murchison and other CM2 carbonaceous chondrites strongly suggest an interstellar heritage for these compounds based on deuterium isotopic anomalies (Pizzarello et al. 1991, 1994; Pizzarello \& Huang 2005). Although a tentative identification of interstellar glycine has been reported, this identification has yet to be verified (Kuan et al. 2003; Snyder et al. 2005). Interstellar amino acids may have a limited lifetime because of low resistance to ultraviolet (UV) photolysis (Ehrenfreund et al. 2001a); amino acid precursors such as nitriles, however, are more stable and may contribute to the population of meteoritic amino acids (Bernstein et al. 2004).

The dominant view of meteoritic amino acid formation has been that these compounds formed via the Strecker synthesis, a reaction in liquid water between hydrogen cyanide ( $\mathrm{HCN})$, ammonia $\left(\mathrm{NH}_{3}\right)$, and an aldehyde ( $\left.\mathrm{RCHO}\right)$. This is presumed to have occurred within meteoritic parent bodies (Peltzer \& Bada 1978; Peltzer et al. 1984; Cronin \& Chang 1993; Cronin et al. 1995; Ehrenfreund et al. 2001b). The isotopic enrichments observed in

\footnotetext{
1 NASA Ames Research Center, Moffett Field, CA 94035-1000; jelsila@ mail.arc.nasa.gov.

2 The SETI Institute, Mountain View, CA 94043.

3 NASA Goddard Space Flight Center, Greenbelt, MD 20771.

4 Catholic University of America, Washington, DC 20064.
}

the meteoritic amino acids are explained by assuming an interstellar heritage for these precursor materials. A Strecker synthesis on the parent body accounts for some observations, such as the recent detection of iminodicarboxylic acids in Murchison (Lerner $\&$ Cooper 2005). However, Strecker synthesis does not explain other observations. For example, the amino acids detected in Murchison are much more enriched in deuterium than their corresponding hydroxy acids (Cronin \& Chang 1993). Conversely, a Strecker synthesis with subsequent deuterium exchange with parent-body water should lead to more deuterium enrichment in the hydroxy acids than in the amino acids, the opposite of what is observed (Lerner 1997). These inconsistencies between the expected Strecker products and the detected meteoritic compounds lead to questions about other potential mechanisms of formation.

These other potential mechanisms include gas-phase formation of interstellar amino acids via ion-molecule reactions, which has been studied both in the laboratory and theoretically (Chakrabarti \& Chakrabarti 2000; Blagovich et al. 2003; Largo et al. 2003). Synthesis of amino acids during molecular cloud collapse has also been proposed (Chakrabarti \& Chakrabarti 2000).

Our focus has been on the promising means of extraterrestrial amino acid formation via energetic processing of interstellar ices, such as those found in dense molecular clouds. Laboratory experiments have shown the synthesis of a variety of amino acids in interstellar ice analogs exposed to UV photolysis (Bernstein et al. 2002; Muñoz Caro et al. 2002) or to electrons mimicking cosmic rays (Holtom et al. 2005). Ice chemistry can produce the type of deuterium enrichments seen in the Murchison amino acids, and the differences between the amino acids and hydroxy acids could be explained by different reaction paths leading to different isotope effects (Sandford et al. 2001). However, the exact mechanism of amino acid formation within these ices has not yet been determined. Suggestions have been made that range from a Strecker-type synthesis (Bernstein et al. 2002) to radicalradical interactions (Sorrell 2001; Woon 2002) to reactions on polycyclic aromatic hydrocarbon flakes within these ices (Mendoza et al. 2004). Complicating the issue, these laboratory and theoretical studies have used several different ice compositions, making it difficult to compare them.

In the work reported here, we examine the formation of amino acids in $\mathrm{UV}$-irradiated ices containing $\mathrm{H}_{2} \mathrm{O}, \mathrm{CH}_{3} \mathrm{OH}, \mathrm{HCN}$, and 
$\mathrm{NH}_{3}$. We use isotopic labeling techniques to illuminate the relationship between the starting materials and the products. We compare these results with theoretical predictions, specifically comparing the observed formation pathway with two mechanisms predicted for this ice composition: the Strecker-type synthesis (Bernstein et al. 2002) and a radical-radical mechanism (Woon 2002). Finally, we discuss the potential connection between these ice-created compounds and meteoritic amino acids and the implications of our observations.

\section{EXPERIMENTAL TECHNIQUE}

\subsection{Ice Preparation}

The interstellar ice analogs were created in an evacuated cryogenic chamber that has been described in detail elsewhere (Allamandola et al. 1988; Bernstein et al. 1995). Two gas mixtures, one typically containing $\mathrm{H}_{2} \mathrm{O}: \mathrm{CH}_{3} \mathrm{OH}: \mathrm{HCN}=20: 2: 2 \mathrm{mbar}$ and the other $\mathrm{H}_{2} \mathrm{O}: \mathrm{CH}_{3} \mathrm{OH}: \mathrm{NH}_{3}=20: 2: 2$ mbar, were vapor deposited simultaneously from two separate glass bulbs onto a cold aluminum foil substrate held at $\sim 20 \mathrm{~K}$. The gas mixtures did not come into contact with one another until immediately before being frozen onto the substrate (Bernstein et al. 2002). The gas mixtures were prepared using $\mathrm{H}_{2} \mathrm{O}$ (purified via a Millipore Milli-Q system to $18.2 \mathrm{M} \Omega$ ) and $\mathrm{CH}_{3} \mathrm{OH}$ (high-precision liquid chromatography [HPLC] grade; Fisher) that were triply freeze-pump-thaw degassed prior to use. $\mathrm{HCN}$ was generated from the addition of concentrated $\mathrm{H}_{2} \mathrm{SO}_{4}$ (Poly Research Corp.) to $\mathrm{KCN}$ (Mallinckrodt) under vacuum, followed by vacuum distillation of the $\mathrm{HCN}$. $\mathrm{NH}_{3}$ was obtained from Mallinckrodt and was used without further purification. $\mathrm{C}$ and/or $\mathrm{N}$ isotopically labeled variants of $\mathrm{CH}_{3} \mathrm{OH}, \mathrm{KCN}$, and $\mathrm{NH}_{3}$ were obtained from Cambridge Isotope Laboratories.

The mixed-molecular ices were photolyzed simultaneously with vapor deposition; photolysis was provided by a microwavepowered flowing-hydrogen discharge lamp (Warneck 1962) that produces an output nearly evenly divided between the Ly $\alpha$ line and a $20 \mathrm{~nm}$ wide molecular transition centered at $160 \mathrm{~nm}$. The lamp was operated at $\sim 100$ mbar of pressure for the $\mathrm{H}_{2}$ gas and with microwave settings of $70 \%$ forward power and $6 \%$ reflected power. The flux of such lamps has been shown to vary with time and operating conditions, but in this work we have assumed a nominal flux of $\sim 2 \times 10^{15}$ photons $\mathrm{cm}^{-2} \mathrm{~s}^{-1}$ (Warneck 1962; Cottin et al. 2003; Loeffler et al. 2005). Typically, the ices were exposed to the equivalent of $\sim 30$ minutes per $0.1 \mu \mathrm{m}$ of ice. This is a reasonable interstellar dose, corresponding to $\sim 500 \mathrm{yr}$ at the edge of a dense could and $\sim 1 \times 10^{7} \mathrm{yr}$ in the interior of such a cloud (Mathis et al. 1983; Prasad \& Tarafdar 1983; Mennella et al. 2003).

After deposition and photolysis, ices were warmed to room temperature at a rate of $2 \mathrm{~K}$ minute $^{-1}$ under dynamic vacuum at $\sim 10^{-8}$ torr. The volatile components of the ice sublimed, leaving an organic residue behind. The foil containing the residue was removed from the vacuum system for analysis as described below. To minimize the potential for contamination, the aluminum foil substrate and all tools and glassware coming into contact with it were pyrolyzed in air in a muffle furnace at $550^{\circ} \mathrm{C}$ overnight prior to use.

\subsection{Sample Analysis}

The organic residue was analyzed by fluorescence detection high-precision liquid chromatography (FD-HPLC) at NASA Ames Research Center, and by fluorescence detection liquid chromatography time-of-flight mass spectrometry (FD-LC/TOF-MS) and gas chromatography mass spectrometry (GC-MS) at NASA
Goddard Space Flight Center. The residue was first sonicated in $500 \mu \mathrm{L}$ Milli-Q water. A portion of this water extract was then acid vapor hydrolyzed (Tsugita et al. 1987; Glavin et al. 2006). After heating, the large test tube was washed, opened, and the hydrolyzed sample inside the smaller test tube was dried and then dissolved again in Milli-Q water for further analysis.

Prior to FD-HPLC or FD-LC/TOF-MS analysis, aliquots of the hydrolyzed samples were fluorescently tagged via derivatization with o-phthalaldehyde/N-acetylcysteine (OPA/NAC; Zhao $\&$ Bada 1995; Glavin et al. 2006). The derivatization reaction was quenched after 1 or 15 minutes at room temperature with either water (NASA Ames) or $75 \mu \mathrm{L}$ of $0.1 \mathrm{M}$ hydrazine hydrate (NASA Goddard).

FD-HPLC at NASA Ames was performed on a $5 \mu \mathrm{L}$ sample injection with a Hewlett-Packard 1100 series HPLC equipped with a fluorescence detector. The separation used a Vydac 218 TP54 column $(4.6 \times 250 \mathrm{~mm}, 5 \mu \mathrm{m}, \mathrm{C} 18)$. The mobile phases were (1) methanol and (2) $50 \mu \mathrm{M}$ sodium acetate ( $\mathrm{pH} 5.4)$ to methanol (92:8); the chromatography protocol of Zhao \& Bada (1995) was used. The HPLC grade methanol, sodium hydroxide, and acetic acid for the HPLC buffer were all obtained from Fisher. Peaks were identified by retention time and comparison with standards. FD-LC/TOF-MS was carried out as described elsewhere (Glavin et al. 2006).

Aliquots of the hydrolyzed samples to be analyzed by GC-MS were treated to convert amino acids to their tert-butyldimethylsilyl derivatives (Mawhinney et al. 1986; MacKenzie et al. 1987; Rodier et al. 2001). Separation was carried out with a Thermo DSQ GC-MS with a splitless injection, an injector temperature of $200^{\circ} \mathrm{C}$ and a flow of $1 \mathrm{~mL}$ minute ${ }^{-1} \mathrm{He}$. A Restek RTX Amine column was used ( $30 \mathrm{~m}, 0.25 \mathrm{~mm}$ ID, $0.5 \mu \mathrm{m}$ film thickness) with a Restek Base Deactivated guard column (5 m). The temperature ramped from 100 to $140^{\circ} \mathrm{C}$ at $20^{\circ} \mathrm{C}$ minute ${ }^{-1}$, was held at $140^{\circ} \mathrm{C}$ for 10 minutes, then was ramped at $1^{\circ} \mathrm{C}$ minute ${ }^{-1}$ to $145^{\circ} \mathrm{C}$, held at $145^{\circ} \mathrm{C}$ for 5 minutes, and finally ramped at $20^{\circ} \mathrm{C}$ minute $^{-1}$ to $250^{\circ} \mathrm{C}$, where it was held for 10 minutes. Masses were recorded from 50 to $650 \mathrm{~m} / \mathrm{z}$ and data analysis was performed using Xcalibur ${ }^{\mathrm{TM}}$ software (Thermo Finnigan).

\subsection{Data Analysis}

The chromatographic data from the FD-LC/TOF-MS data were used to identify the amino acids and to calculate product yields. The mass spectral data were used qualitatively to identify the major mass peaks for each amino acid and to determine the general number of labeled carbon or nitrogen atoms in each species.

Peak intensities for the measured GC-MS masses were used to calculate relative mass abundances. The expected peak distributions for derivatized amino acids containing various numbers of labeled carbon or nitrogen atoms were also calculated. A least-squares fit performed on the relative peak area data using MATLAB ${ }^{\circledR}$ (Mathworks) was used to derive the approximate contribution from each labeled species. Because the uncertainties from background interferences and from the mathematical fit are difficult to quantify, we do not report the calculated contributions quantitatively here. Rather, we use these calculations to report in a more general manner on the observed isotopic patterns and their implications in elucidating the reaction mechanisms.

\section{RESULTS}

\subsection{Amino Acid Production from Various Ice Compositions}

We began our studies with UV photolysis of an interstellar ice analog with composition $\mathrm{H}_{2} \mathrm{O}: \mathrm{CH}_{3} \mathrm{OH}: \mathrm{HCN}: \mathrm{NH}_{3}=20: 2$ : $1: 1$. This composition was chosen for three reasons: (1) it was 


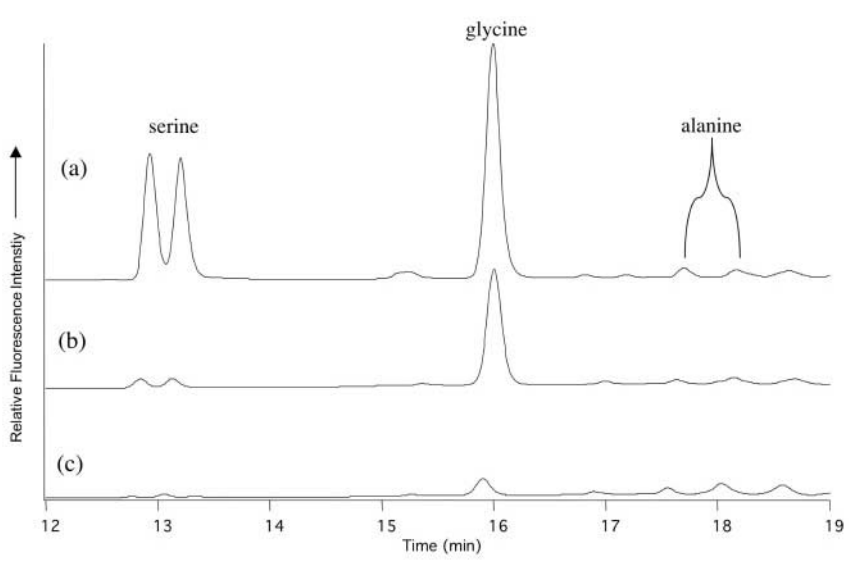

FIG. 1.-FD-HPLC traces of hydrolyzed derivatized residues from ices with composition $(a) \mathrm{H}_{2} \mathrm{O}: \mathrm{CH}_{3} \mathrm{OH}: \mathrm{HCN}: \mathrm{NH}_{3}=20: 2: 1: 1 ;(b) \mathrm{H}_{2} \mathrm{O}$ : $\mathrm{CH}_{3} \mathrm{OH}: \mathrm{HCN}=20: 2: 1$; and (c) $\mathrm{H}_{2} \mathrm{O}: \mathrm{CH}_{3} \mathrm{OH}: \mathrm{NH}_{3}=20: 2: 1$. The presence of prominent glycine and serine peaks in $(a)$ and $(b)$ but not in $(c)$ suggests that $\mathrm{HCN}$ is more important for formation of these amino acids than is $\mathrm{NH}_{3}$.

previously studied both in the laboratory and theoretically (Bernstein et al. 2002; Woon 2002); (2) this composition matches that required for a potential Strecker-type synthesis; and (3) these are reasonable interstellar relative abundances. $\mathrm{H}_{2} \mathrm{O}, \mathrm{CH}_{3} \mathrm{OH}$, and $\mathrm{NH}_{3}$ are among the most abundant molecules identified in frozen grains in the dense interstellar medium (ISM; Allamandola et al. 1992; Lacy et al. 1998), while HCN, although it has not yet been detected in icy grains, is also abundant in the gas phase in the dense ISM (Snyder \& Buhl 1971; Boonman et al. 2001) and should be efficiently condensed onto grain surfaces in cold regions of dense clouds. As shown in Figure $1 a$, the major product observed on photolysis of this ice was glycine, with measurable amounts of racemic serine and alanine, as well as some minor products. The structures of these three amino acids are depicted in Figure 2. FD-LC/TOF-MS and GC-MS analyses of these ice residues (not shown) confirmed these results, and also identified a variety of minor products. The following products were identified in these hydrolyzed residues (yield relative to glycine shown in parentheses): glycine (100), D-serine (58), L-serine (56), D-alanine (3), L-alanine (2), $\beta$-alanine (1), ethanolamine (225), and methylamine (7). Yields were calculated as described in Glavin et al. (2006). As in the previous studies, the racemic nature of the amino acids produced, as well as analysis of control experiments (not shown), verified that these compounds are produced by the UV photolysis and are not contaminants.

To determine the importance of $\mathrm{HCN}$ and $\mathrm{NH}_{3}$ in formation of these amino acids, we photolyzed ices in which one of these components was missing. The FD-HPLC traces of the resulting ice residues are shown in Figures $1 b$ and $1 c$. In the $\mathrm{H}_{2} \mathrm{O}: \mathrm{CH}_{3} \mathrm{OH}$ : $\mathrm{HCN}=20: 2: 1$ ice (no $\mathrm{NH}_{3}$; Fig. $1 b$ ), measurable quantities of glycine, racemic serine, and racemic alanine were again produced. The absolute yield of glycine was approximately $80 \%$ of its yield compared to the ice containing all four components; the yield of serine relative to glycine dropped to $\sim 5 \%$, and the relative yield of alanine remained $\sim 5 \%$. In the $\mathrm{H}_{2} \mathrm{O}: \mathrm{CH}_{3} \mathrm{OH}$ : $\mathrm{NH}_{3}=20: 2: 1$ ice (no HCN; Fig. 1c), the overall yield of all amino acids was greatly reduced. Glycine was produced at approximately $10 \%$ of the yield seen in the ice containing all four components, while serine was undetectable. The absolute yield of alanine was slightly higher than its yield in the ice containing

\section{Glycine}

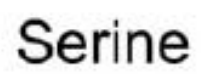

Alanine

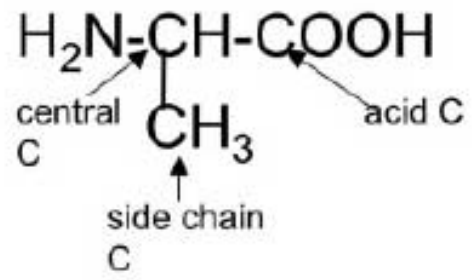

FIG. 2.- Structures of the amino acids glycine, serine, and alanine. The carbon atoms are labeled to differentiate them.

all four components, giving it a yield of $\sim 75 \%$ relative to glycine in this ice.

We also performed reactions where ethanol was substituted for methanol, with an ice composition of $\mathrm{H}_{2} \mathrm{O}: \mathrm{C}_{2} \mathrm{H}_{5} \mathrm{OH}: \mathrm{HCN}$ : $\mathrm{NH}_{3}=20: 2: 1: 1$. Overall amino acid yield was greatly decreased, with glycine (the major product) produced at $\sim 10 \%$ of its yield in the methanol-containing ice. However, a wider range of amino acids was formed. These hydrolyzed ice residues produced the following compounds (with their yields relative to glycine in parentheses): glycine (100), D,L-alanine (26), $\beta$-alanine (5), D,L-serine (14), D,L-threonine (60), D,L-allothreonine (30), D, L-homoserine (1), D,L- $\alpha$-amino-n-butyric acid (1), D,L- $\beta$-aminon-butyric acid (4), $\gamma$-amino-n-butyric acid (2), methylamine (8), and ethanolamine (12).

\subsection{Isotope Labeling}

We used isotopic labeling of the compounds in our ice mixtures to examine the pathway from reagents to products in these ices. We carried out both ${ }^{13} \mathrm{C}$ and ${ }^{15} \mathrm{~N}$ labeling experiments. Our FD-LC/TOF-MS analyses of these ice residues provided sensitive information about the mass of each amino acid produced, allowing the determination of the presence or absence of labeled atoms in the product molecule. GC-MS measurements verified the FD-LC/TOF-MS data on the whole product molecules and also included fragment masses for the most abundant compounds, which allowed us to determine the location of the labeled atom in the molecule and thus the relative contribution of each starting material to each individual carbon and nitrogen atom. Results were compared with analyses of standard samples and with calculations of expected mass distributions. As described in $\S 2.3$, although we used quantitative methods to derive relative contributions of each labeled species, we discuss the results somewhat more qualitatively because of the associated uncertainties with the technique. 


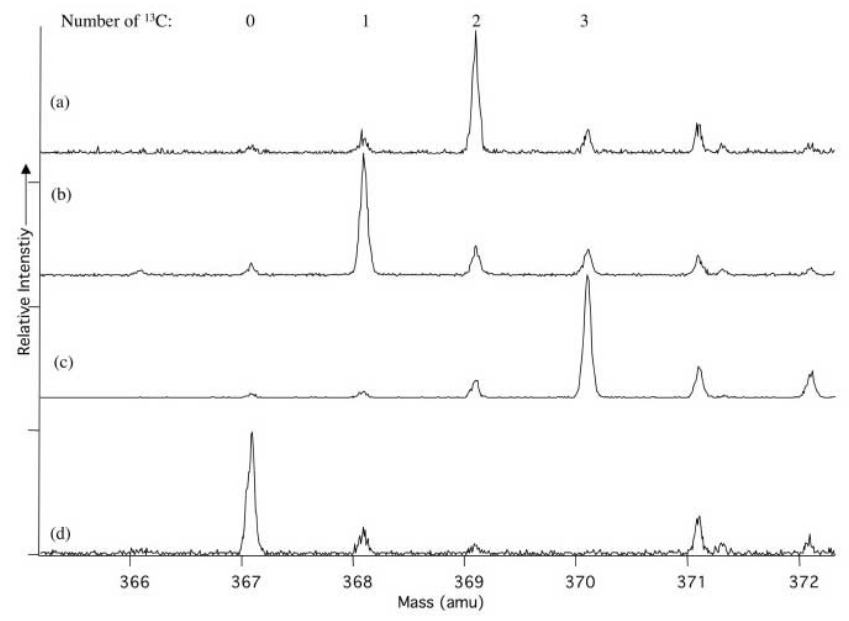

FIG. 3.-Positive electrospray FD-LC/TOF-MS spectra of OPA/NAC derivatized acid-hydrolyzed extracts of $(a)$ residue from an ice with composition $\mathrm{H}_{2} \mathrm{O}$ : $\mathrm{CH}_{3} \mathrm{OH}: \mathrm{H}^{13} \mathrm{CN}: \mathrm{NH}_{3}=20: 2: 1: 1 ;(b)$ residue from an ice with composition $\mathrm{H}_{2} \mathrm{O}:{ }^{13} \mathrm{CH}_{3} \mathrm{OH}: \mathrm{HCN}: \mathrm{NH}_{3}=20: 2: 1: 1 ;(c)$ residue from an ice with composition $\mathrm{H}_{2} \mathrm{O}:{ }^{13} \mathrm{CH}_{3} \mathrm{OH}: \mathrm{H}^{13} \mathrm{CN}: \mathrm{NH}_{3}=20: 2: 1: 1$; and $(d)$ a serine standard. The peak at 367 amu represents unlabeled serine, while peaks at higher masses indicate the incorporation of one or more ${ }^{13} \mathrm{C}$ atoms. The peaks at 371 and $372 \mathrm{amu}$ are interfering contaminant peaks. Note that the OPA/NAC fluorescent label provides terrestrial ${ }^{13} \mathrm{C}$.

\subsubsection{Serine}

Although serine is not the simplest amino acid structurally, it produced the most straightforward isotopic results, so we present its data first. Figure 3 shows the positive electrospray FD-LC/ TOF-MS spectra of derivatized aliquots of the hydrolyzed residues from three ices with various isotopically labeled components (Figs. $3 a-3 c$ ) and a serine standard (Fig. $3 d$ ).

Serine contains three carbon atoms: an acid carbon, a central carbon, and a side-chain carbon (see structure in Fig. 2). Its OPA/NAC derivative detected by positive electrospray FD-LC/ TOF-MS has a nominal mass of $367 \mathrm{amu}$ (OPA/NAC-Ala$\mathrm{H}^{+}=\mathrm{C}_{13} \mathrm{H}_{13} \mathrm{NO}_{3} \mathrm{~S}-\mathrm{C}_{3} \mathrm{H}_{5} \mathrm{NO}_{3}-\mathrm{H}^{+}$) (as seen in Fig. $3 d$ ). The smaller peaks at higher masses are caused by naturally occurring isotope distributions from both the three serine carbon atoms and the carbon atoms added by the OPA/NAC derivatizing agent. Figure $3 c$ shows that when only ${ }^{13} \mathrm{C}$ isotopically labeled starting materials are present in the ice $\left({ }^{13} \mathrm{CH}_{3} \mathrm{OH}\right.$ and $\left.\mathrm{H}^{13} \mathrm{CN}\right)$, the detected nominal mass is at $370 \mathrm{amu}\left(\mathrm{C}_{13} \mathrm{H}_{13} \mathrm{NO}_{3} \mathrm{~S}-{ }^{13} \mathrm{C}_{3} \mathrm{H}_{5} \mathrm{NO}_{3}-\mathrm{H}^{+}\right)$. This mass shift indicates the incorporation of three ${ }^{13} \mathrm{C}$ atoms into the serine, demonstrating that all the detected serine was produced from our labeled reagents and was not contamination.

In Figure $3 a$, we see that when labeled $\mathrm{H}^{13} \mathrm{CN}$ is used, the major peak shifts to a mass of $369 \mathrm{amu}$. This shift indicates that two labeled carbon atoms have been incorporated into the serine. Conversely, in Figure $3 b$, we see that the use of labeled ${ }^{13} \mathrm{CH}_{3} \mathrm{OH}$ produces a serine derivative with mass $368 \mathrm{amu}$, indicating that one labeled carbon atom has been incorporated. Taken together, these results strongly suggest that two of serine's carbon atoms derive predominantly from the $\mathrm{HCN}$ in the ice, with the third carbon atom generally originating from the $\mathrm{CH}_{3} \mathrm{OH}$.

GC-MS analysis of these ice residues allowed us to pinpoint the origin of each of the carbon atoms in the serine. GC-MS provides mass information for the unfragmented serine ion and for three main fragments. Two of these fragments result from the loss of the acid carbon, while the third results from the loss of the sidechain carbon (Mawhinney et al. 1986). We compared the peak distribution for each mass measured with the calculated isotopic

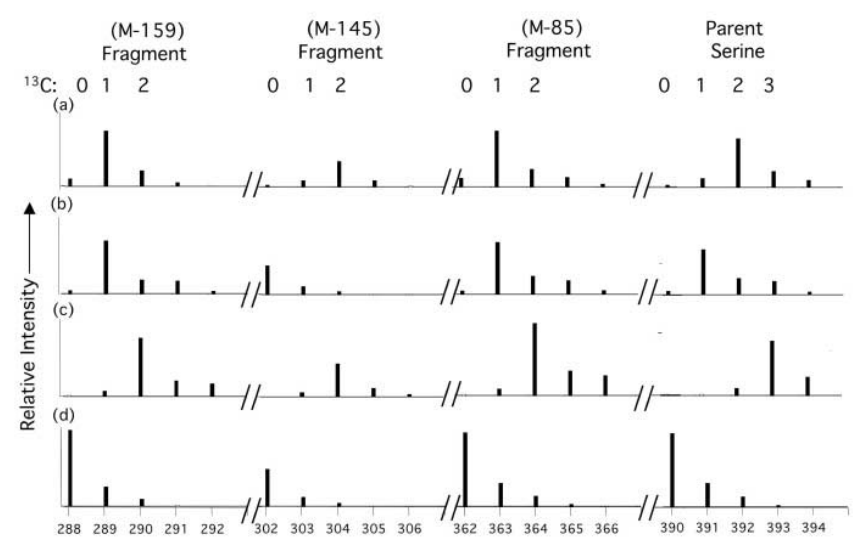

FIG. 4.-GC-MS mass chromatograms for serine and its main fragment masses for the tert-butlydimethylsilyl derivatized acid-hydrolyzed extracts of (a) residue from an ice with composition $\mathrm{H}_{2} \mathrm{O}: \mathrm{CH}_{3} \mathrm{OH}: \mathrm{H}^{13} \mathrm{CN}: \mathrm{NH}_{3}=20$ : $2: 1: 1 ;(b)$ residue from an ice with composition $\mathrm{H}_{2} \mathrm{O}:{ }^{13} \mathrm{CH}_{3} \mathrm{OH}: \mathrm{HCN}: \mathrm{NH}_{3}=$ $20: 2: 1: 1 ;(c)$ residue from an ice with composition $\mathrm{H}_{2} \mathrm{O}:{ }^{13} \mathrm{CH}_{3} \mathrm{OH}: \mathrm{H}^{13} \mathrm{CN}$ : $\mathrm{NH}_{3}=20: 2: 1: 1$; and $(d)$ a serine standard. The peak at 390 amu represents the parent serine ion containing the central carbon, acid carbon, and side carbon. The fragments at $362 \mathrm{amu}(\mathrm{M}-85)$ and $288 \mathrm{amu}(\mathrm{M}-159)$ reflect the loss of the acid carbon. The fragment at 302 amu (M-145) reflects the loss of the side-chain carbon.

distributions to determine the contribution of labeled atoms to each mass. Within our experimental uncertainties, we observed contributions from only one major reaction pathway for the formation of serine.

The GC-MS data is shown in Figure 4 and summarized in Table 1 and correlate well with the FD-LC/TOF-MS data. For the derivatized major serine ion (M-57; loss of a t-butyl group from one MTBS group) $\left[\left(\mathrm{C}_{6} \mathrm{H}_{15} \mathrm{Si}\right)_{2}\left(\mathrm{C}_{2} \mathrm{H}_{7} \mathrm{Si}\right)-\mathrm{C}_{3} \mathrm{H}_{3} \mathrm{NO}_{3}^{+}\right.$mass $=$ $390 \mathrm{amu}$, the residue from the ice containing ${ }^{13} \mathrm{CH}_{3} \mathrm{OH}$ and $\mathrm{H}^{13} \mathrm{CN}$ (ice 3; Fig. 4c) showed primarily three labeled carbon atoms, while the serine standard (Fig. $4 d$ ) contained no labeled carbon atoms. The residue from the $\mathrm{H}^{13} \mathrm{CN}$-containing ice (ice 1; Fig. $4 a$ ) revealed predominantly serine with two labeled atoms, while the residue from the ${ }^{13} \mathrm{CH}_{3} \mathrm{OH}$-containing ice (ice 2; Fig. $4 c$ ) showed serine containing primarily one labeled carbon atom.

The two fragments that result from the loss of the acid carbon (M-85 and M-159) showed similar results; in both of these, the fragment of serine resulting from the $\mathrm{H}^{13} \mathrm{CN}$ ice (ice 1; Fig. 4a) contained primarily one labeled carbon atom, as did the residue from the ${ }^{13} \mathrm{CH}_{3} \mathrm{OH}$ ice (ice 2; Fig. $4 b$ ). Since we know that the unfragmented serine contained two carbon atoms from $\mathrm{HCN}$ and one from $\mathrm{CH}_{3} \mathrm{OH}$, the lost acid carbon atom must therefore originate from the $\mathrm{HCN}$.

The side-chain carbon in serine is analyzed via a GC-MS fragment that results from the loss of this carbon (M-145). This fragment showed two labeled $\mathrm{C}$ atoms in the ice containing $\mathrm{H}^{13} \mathrm{CN}$ (ice 1; Fig. $4 a$ ) and no labeled carbon atoms in the ice containing ${ }^{13} \mathrm{CH}_{3} \mathrm{OH}$ (ice 2; Fig. 4b). As the analysis of the unfragmented serine showed a contribution of two carbon atoms from $\mathrm{HCN}$ and one from $\mathrm{CH}_{3} \mathrm{OH}$, these observations suggest that the lost side-chain carbon originates from the ${ }^{13} \mathrm{CH}_{3} \mathrm{OH}$, and therefore, the central carbon atom must originate from $\mathrm{HCN}$.

In our ${ }^{15} \mathrm{~N}$-labeling experiments (GC-MS not shown), all of the available carbon atoms were labeled in order to be certain that observed products were not the result of contamination. The control experiment (ice 6), in which all of the nitrogen atoms in the starting material were also labeled, produced only serine with $a^{15} \mathrm{~N}$ atom (see Table 1). In the ices containing $\mathrm{H}^{13} \mathrm{C}^{15} \mathrm{~N}$ (ices 4 and 7), a majority of the serine produced contained a ${ }^{15} \mathrm{~N}$ atom. 
TABLE 1

Summary of Serine Isotopic GC-MS Labeling Results for the Observed Major Product

\begin{tabular}{|c|c|c|c|c|c|c|c|c|c|}
\hline \multirow[b]{2}{*}{ ICE } & \multirow[b]{2}{*}{$\mathrm{CH}_{3} \mathrm{OH}^{\mathrm{a}}$} & \multirow[b]{2}{*}{$\mathrm{HCN}^{\mathrm{a}}$} & \multirow[b]{2}{*}{$\mathrm{NH}_{3}{ }^{\mathrm{a}}$} & \multicolumn{2}{|c|}{ (M-57) Parent SERINE ${ }^{\mathrm{b}}$} & \multicolumn{2}{|c|}{$(\mathrm{M}-85)$ AND (M-159) FraGMENTS ${ }^{\mathrm{c}}$} & \multicolumn{2}{|c|}{$(\mathrm{M}-145)$ Fragment $^{\mathrm{d}}$} \\
\hline & & & & Labeled C Atoms & Labeled N Atoms & Labeled C Atoms & Labeled N Atoms & Labeled C Atoms & Labeled N Atoms \\
\hline $2 \ldots \ldots$ & ${ }^{13} \mathrm{C}$ & & & 1 & 0 & 1 & 0 & 0 & 0 \\
\hline 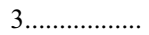 & ${ }^{13} \mathrm{C}$ & ${ }^{13} \mathrm{C}$ & & 3 & 0 & 2 & 0 & 2 & 0 \\
\hline $4 \ldots \ldots \ldots \ldots \ldots$ & ${ }^{13} \mathrm{C}$ & ${ }^{13} \mathrm{C},{ }^{15} \mathrm{~N}$ & & 3 & 1 & 2 & 1 & 2 & 1 \\
\hline $5 \ldots \ldots \ldots \ldots \ldots$ & ${ }^{13} \mathrm{C}$ & ${ }^{13} \mathrm{C}$ & ${ }^{15} \mathrm{~N}$ & 3 & 0 or $1^{\mathrm{e}}$ & ${ }^{f}{ }^{f}$ & $\ldots$ & $\ldots$ & $\ldots$ \\
\hline
\end{tabular}

${ }^{a}$ All ices had a composition of $\mathrm{H}_{2} \mathrm{O}: \mathrm{CH}_{3} \mathrm{OH}: \mathrm{HCN}: \mathrm{NH}_{3}=20: 2: 1: 1$. The isotopically labeled variants of these components are indicated here; blank entries indicate that the species used in that ice contained terrestrial isotopic ratios.

${ }^{\mathrm{b}}$ Serine contains three carbon atoms and one nitrogen atom.

c These two fragments originate from the loss of the acid carbon; the remaining fragment contains two carbon atoms and one nitrogen atom.

d This fragment originates from the loss of the side-chain carbon; the remaining fragment contains two carbon atoms and one nitrogen atom.

e In this ice, approximately $5 \%$ of the serine molecules contained a labeled nitrogen atom.

${ }^{\mathrm{f}}$ No entries are shown for fragmentation in these ices, because no information is gained from these fragments.

In the ice containing ${ }^{15} \mathrm{NH}_{3}$ (ice 5) only a small fraction of the produced serine contained $\mathrm{a}^{15} \mathrm{~N}$ atom. Our analysis suggests that the nitrogen atom arises from $\mathrm{NH}_{3}$ only $\sim 5 \%$ of the time, with the remaining serine receiving its nitrogen from HCN. Fragmentation patterns are not necessary for analysis of this data, as there is only one nitrogen atom in serine. These results indicate that the majority, but not all, of the produced serine received its nitrogen atom from the $\mathrm{HCN}$ molecule, not the $\mathrm{NH}_{3}$.

Combining all of this data suggests that the majority of the serine made in our ices receives its central carbon, its acid carbon, and its nitrogen atom from the $\mathrm{HCN}$, while the $\mathrm{CH}_{3} \mathrm{OH}$ provides its side-chain carbon.

\subsubsection{Glycine}

Results from analysis of glycine in our ice residues is presented in Figure 5 and summarized in Table 2. Figure 5 shows the positive electrospray FD-LC/TOF-MS spectra of derivatized aliquots of the hydrolyzed ice residues from ices 1 (Fig. $5 a$ ), 2 (Fig. 5b), 3 (Fig. 5c), and a glycine standard (Fig. 5d). Glycine

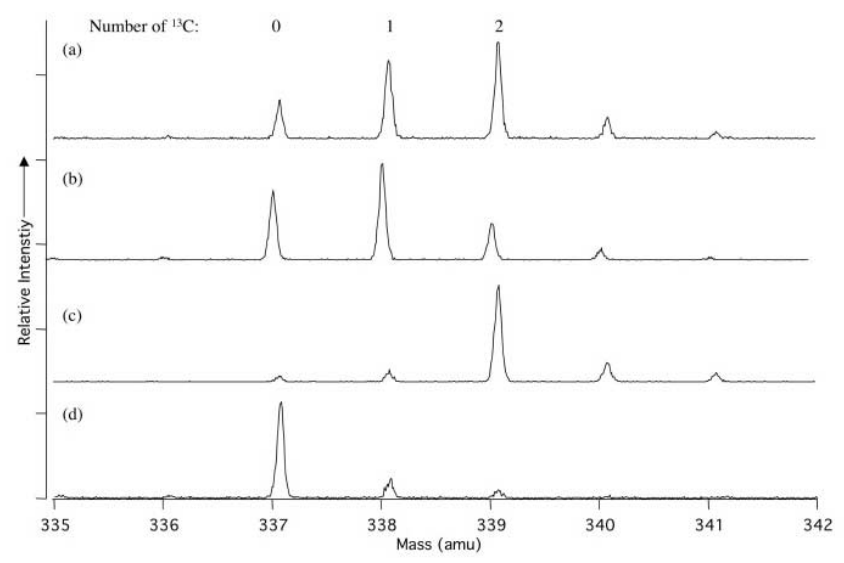

FIG. 5.-Positive electrospray FD-LC/TOF-MS spectra of OPA/NAC derivatized acid-hydrolyzed extracts of $(a)$ residue from an ice with composition $\mathrm{H}_{2} \mathrm{O}: \mathrm{CH}_{3} \mathrm{OH}: \mathrm{H}^{13} \mathrm{CN}: \mathrm{NH}_{3}=20: 2: 1: 1 ;(b)$ residue from an ice with composition $\mathrm{H}_{2} \mathrm{O}:{ }^{13} \mathrm{CH}_{3} \mathrm{OH}: \mathrm{HCN}: \mathrm{NH}_{3}=20: 2: 1: 1$; (c) residue from an ice with composition $\mathrm{H}_{2} \mathrm{O}:{ }^{13} \mathrm{CH}_{3} \mathrm{OH}: \mathrm{H}^{13} \mathrm{CN}: \mathrm{NH}_{3}=20: 2: 1: 1$; and (d) a glycine standard. The peak at 337 amu represents unlabeled glycine, while peaks at higher masses indicate the incorporation of one or more ${ }^{13} \mathrm{C}$ atoms. Note that the OPA/NAC fluorescent label provides terrestrial ${ }^{13} \mathrm{C}$. contains two carbon atoms: an acid carbon and a central carbon (see structure in Fig. 2). The glycine standard (Fig. 5d) showed a single peak at a nominal mass of $337 \mathrm{amu}$, representing the positive ion of the OPA/NAC derivatized glycine compound $\left(\mathrm{C}_{13} \mathrm{H}_{13} \mathrm{NO}_{3} \mathrm{~S}-\mathrm{C}_{2} \mathrm{H}_{3} \mathrm{NO}_{2}-\mathrm{H}^{+}\right)$. The spectrum of glycine from the ${ }^{13} \mathrm{CH}_{3} \mathrm{OH}$ and $\mathrm{H}^{13} \mathrm{CN}$-containing ice (ice 3; Fig. $5 c$ ) showed this peak shifted to $339 \mathrm{amu}$, indicating that both carbon atoms were isotopically labeled.

Analysis of the ice containing $\mathrm{H}^{13} \mathrm{CN}$ (ice 1; Figure 5a) revealed a mix of masses in the glycine, with the major peaks at 338 and 339 amu and a minor peak at 337 amu. This mix indicates that the glycine produced in this ice usually incorporated one or two carbon atoms from the $\mathrm{H}^{13} \mathrm{CN}$, with a small amount of glycine produced containing no carbon from HCN. By comparison, in Figure $5 b$, we see that the use of labeled ${ }^{13} \mathrm{CH}_{3} \mathrm{OH}$ (ice 2) produces glycine derivatives primarily with mass 337 and 338 , indicating that the glycine tends to incorporate either zero or one carbon atoms from the ${ }^{13} \mathrm{CH}_{3} \mathrm{OH}$. A minor peak at mass $339 \mathrm{amu}$ indicates that some glycine may be produced with two carbon atoms contributed by $\mathrm{CH}_{3} \mathrm{OH}$.

The GC-MS data from these experiments are summarized in Table 2 and again correlate well with the FD-LC/TOF-MS data. For the derivatized major glycine ion (M-57; loss of a t-butyl group from one MTBS group) $\left(\mathrm{C}_{6} \mathrm{H}_{15} \mathrm{Si}\right)\left(\mathrm{C}_{2} \mathrm{H}_{7} \mathrm{Si}\right)-\mathrm{C}_{2} \mathrm{H}_{2} \mathrm{NO}_{2}^{+}$mass $=$ $246 \mathrm{amu}$ ), the residue from the ice containing ${ }^{13} \mathrm{CH}_{3} \mathrm{OH}$ and $\mathrm{H}^{13} \mathrm{CN}$ (ice 3) showed two labeled carbon atoms, as expected. The residue from the ${ }^{13} \mathrm{CH}_{3} \mathrm{OH}$-containing ice (ice 2) revealed approximately equal amounts of glycine containing zero labeled atoms $(\sim 40 \%)$ and one labeled atom $(\sim 50 \%)$, with a small amount $(\sim 10 \%)$ containing two labeled carbon atoms. The residue from the $\mathrm{H}^{13} \mathrm{CN}$ ice (ice 1) showed equal amounts of glycine with either one or two labeled atoms; no unlabeled glycine was observed.

The fragment resulting from loss of the acid carbon (M-85) has a mass of $218 \mathrm{amu}$ and contains the central carbon atom. In the residue from the ${ }^{13} \mathrm{CH}_{3} \mathrm{OH}$ and $\mathrm{H}^{13} \mathrm{CN}$-containing ice (ice 3), this carbon was fully labeled. In the ${ }^{13} \mathrm{CH}_{3} \mathrm{OH}$-containing ice (ice 2), we again saw approximately equal amounts of the $\mathrm{H}_{2} \mathrm{~N}-\mathrm{CH}_{2}$ fragment of glycine containing zero or one labeled carbon atoms. The residue from the $\mathrm{H}^{13} \mathrm{CN}$ ice (ice 1) also showed approximately equal amounts of either zero or one labeled carbon atoms in this fragment.

Combining the results from the fragmented and whole glycine, it appears that the glycine produced in this ice contains either zero 
TABLE 2

Summary of Glycine GC-MS Isotopic Labeling Results

\begin{tabular}{|c|c|c|c|c|c|c|c|}
\hline ICE & $\mathrm{CH}_{3} \mathrm{OH}^{\mathrm{a}}$ & $\mathrm{HCN}^{\mathrm{a}}$ & $\mathrm{NH}_{3}{ }^{\mathrm{a}}$ & \multicolumn{2}{|c|}{ UNFRAGMENTED GLYCINE $^{\mathrm{b}}$} & \multicolumn{2}{|c|}{$(\mathrm{M}-85)$ FRAGMENT $^{\mathrm{c}}$} \\
\hline 1 & & ${ }^{13} \mathrm{C}$ & & 1 or 2 & 0 & 0 or 1 & 0 \\
\hline $2 \ldots \ldots \ldots \ldots \ldots \ldots \ldots \ldots$ & ${ }^{13} \mathrm{C}$ & & & 0,1, or $2^{\mathrm{d}}$ & 0 & 0 or 1 & 0 \\
\hline 3.․․․․․․․․… & ${ }^{13} \mathrm{C}$ & ${ }^{13} \mathrm{C}$ & & 2 & 0 & 1 & 0 \\
\hline 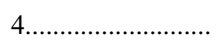 & ${ }^{13} \mathrm{C}$ & ${ }^{13} \mathrm{C},{ }^{15} \mathrm{~N}$ & & 0,1, or $2^{\mathrm{d}}$ & 1 & 0 or 1 & 1 \\
\hline $5 \ldots \ldots \ldots \ldots \ldots \ldots$ & ${ }^{13} \mathrm{C}$ & ${ }^{13} \mathrm{C}$ & ${ }^{15} \mathrm{~N}$ & 2 & 0 or 1 & $\ldots{ }^{\mathrm{e}}$ & $\ldots$ \\
\hline
\end{tabular}

${ }^{\text {a }}$ All ices had a composition of $\mathrm{H}_{2} \mathrm{O}: \mathrm{CH}_{3} \mathrm{OH}: \mathrm{HCN}: \mathrm{NH}_{3}=20: 2: 1: 1$. The isotopically labeled variants of these components are indicated here; blank entries indicate that the species used in that ice contained terrestrial isotopic ratios.

${ }^{\mathrm{b}}$ Glycine contains two carbon atoms and one nitrogen atom.

c This fragment originates from the loss of the acid carbon; the remaining fragment contains one carbon atom and one nitrogen atom.

d Approximately $10 \%$ of the double label was observed, with the remainder equally distributed between zero and one labeled atoms.

${ }^{\mathrm{e}}$ No entries are shown for fragmentation in these ices, because no information is gained from these fragments.

or one atoms from $\mathrm{CH}_{3} \mathrm{OH}$ and one or two $\mathrm{C}$ atoms from $\mathrm{HCN}$. The acid carbon appears to derive almost entirely from the HCN, which means that the central carbon can arise from either the $\mathrm{CH}_{3} \mathrm{OH}(\sim 50 \%)$ or the $\mathrm{HCN}(\sim 50 \%)$. A small amount of glycine is produced that contains both carbon atoms from the $\mathrm{CH}_{3} \mathrm{OH}$.

In our ${ }^{15} \mathrm{~N}$-labeling experiments, the control (ice 6) produced fully labeled glycine, as expected. In the ice containing ${ }^{15} \mathrm{NH}_{3}$ (ice 5), the majority of the glycine was not labeled at the nitrogen atom; only $\sim 10 \%$ showed the presence of a labeled ${ }^{15} \mathrm{~N}$ atom. In the $\mathrm{H}^{13} \mathrm{C}^{15} \mathrm{~N}$-containing ice (ice 4), these ratios were reversed, with the majority of the glycine containing a ${ }^{15} \mathrm{~N}$ atom. These results indicate that the majority, but not all, of the glycine received its nitrogen atom from the $\mathrm{HCN}$ molecule, not the $\mathrm{NH}_{3}$.

Finally, ice 7 examined the $\mathrm{C} \equiv \mathrm{N}$ moiety in the $\mathrm{HCN}$ starting material by using $\mathrm{H}^{13} \mathrm{C}^{15} \mathrm{~N}$. By labeling both the $\mathrm{C}$ and $\mathrm{N}$ atoms and using GC-MS analysis of the 218 fragment, we could trace the $\mathrm{C} \equiv \mathrm{N}$ bond and determine if these two atoms remained together in the product). Both the FD-LC/TOF-MS and GC-MS data (not shown) indicated that $\sim 60 \%$ of the glycine was fully labeled; that is, both carbon atoms and the nitrogen atom derived from the $\mathrm{HCN}$. In these cases, the $\mathrm{C} \equiv \mathrm{N}$ moiety remained intact, forming the $\mathrm{H}_{2} \mathrm{~N}-\mathrm{CH}_{2}$ portion of the glycine. In the remainder of the glycine molecules, the original $\mathrm{C} \equiv \mathrm{N}$ bond broke and glycine was formed with a $\mathrm{CH}_{3} \mathrm{OH}$-derived central carbon and with nitrogen from either $\mathrm{HCN}$ or $\mathrm{NH}_{3}$.

\subsubsection{Other Compounds}

Alanine was produced in these ices at much lower levels than glycine and serine, making it more difficult to analyze. Alanine, like serine, contains three carbon atoms (see structure in Fig. 2): an acid carbon, a central carbon, and a side-chain carbon. In our carbon-labeling experiments, we observed a contribution to the alanine of one or two carbon atoms from both the $\mathrm{CH}_{3} \mathrm{OH}$ and the $\mathrm{HCN}$, indicating that both compounds participate in the formation of alanine. The control experiment, in which both $\mathrm{CH}_{3} \mathrm{OH}$ and $\mathrm{HCN}$ were labeled, showed a negligible amount of contamination. GC-MS fragmentation analysis was more difficult for these experiments because of the low concentration of alanine, and no reliable information could be obtained from fragmentation.

For the nitrogen-labeled experiments, only FD-LC/TOF-MS analysis could be carried out; insufficient alanine was produced for GC-MS analysis. The results suggest that $\mathrm{HCN}$ provides the nitrogen in the majority of alanine produced $(\sim 65 \%)$, with the remaining 35\% receiving its nitrogen from $\mathrm{NH}_{3}$.
Other minor compounds that we observed included $\beta$-alanine (an isomer of alanine), methylamine $\left(\mathrm{CH}_{3} \mathrm{NH}_{2}\right)$, and ethanolamine $\left(\mathrm{HOCH}_{2} \mathrm{CH}_{2} \mathrm{NH}_{2}\right)$. The FD-LC/TOF-MS analyses of each of these compounds showed a variety of pathways. For $\beta$-alanine, it appeared that $\mathrm{CH}_{3} \mathrm{OH}$ contributed up to three carbons, while $\mathrm{HCN}$ contributed up to two; the majority of the nitrogen arose from $\mathrm{HCN}$. These results are similar to the results for alanine. The methylamine showed contributions of carbon from either $\mathrm{CH}_{3} \mathrm{OH}$ and $\mathrm{HCN}$ and nitrogen from either $\mathrm{HCN}$ or $\mathrm{NH}_{3}$, with a slight preference toward the HCN. Ethanolamine gave the clearest results for these minor compounds; it derived one carbon each from $\mathrm{HCN}$ and $\mathrm{CH}_{3} \mathrm{OH}$, and its nitrogen arose from $\mathrm{HCN}$.

\section{DISCUSSION}

\subsection{Comparison with Predictions}

Based on the results of the labeling experiments described above, we were able to determine the origin of the carbon and nitrogen atoms in glycine and serine produced in these interstellar ice analogs. Figure $6 a$ summarizes these results.

We observe that although there is one dominant pathway in the formation of each of these amino acids, minor reaction paths exist as well. In both amino acids, the major pathway results from $\mathrm{HCN}$ contributing the nitrogen, central carbon, and acid carbon atoms. For serine, the side-chain carbon arises from $\mathrm{CH}_{3} \mathrm{OH}$. Glycine shows more contribution from several minor pathways in which the nitrogen can be provided by $\mathrm{NH}_{3}$ and/or the central carbon is provided by $\mathrm{CH}_{3} \mathrm{OH}$. Serine has a small contribution from a pathway in which $\mathrm{NH}_{3}$ provides the nitrogen, but the origin of the carbon atoms does not appear to change. It is plausible that the photochemical conversion of $\mathrm{NH}_{3}$ to $\mathrm{HCN}$ or one of its reaction products is responsible for this observation (Palumbo et al. 2000; Wu et al. 2005). For the minor products (alanine, $\beta$-alanine, methylamine, ethanolamine), there appear to be a variety of mechanisms as well, although we were unable to determine them exactly.

Our observed results for glycine correlate well with the experiments in which either $\mathrm{HCN}$ or $\mathrm{NH}_{3}$ were absent from the ices (see Fig. 1 above). With $\mathrm{NH}_{3}$ absent, glycine was still produced in large amounts; without $\mathrm{HCN}$, on the other hand, glycine was produced, but yields were reduced dramatically. The change in yields is likely due to the different efficiencies of the major versus minor pathways. The serine isotopic labeling results also correlate with these experiments; serine was undetectable in the absence of $\mathrm{HCN}$, but was produced (although in low yields) in the absence of 
(a) $\mathrm{H}_{2} \mathrm{O}+\mathrm{CH}_{3} \mathrm{OH}+\mathrm{HCN}+\mathrm{NH}_{3} \stackrel{\text { UV photolysis }}{\longrightarrow}$

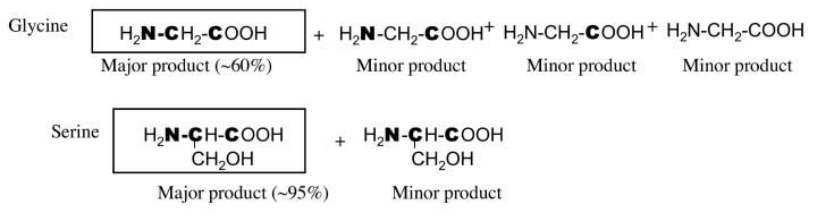

(b)

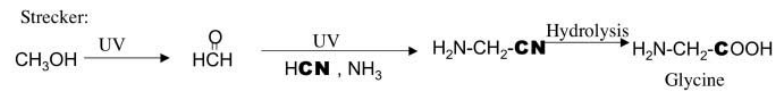

Radical-radical:

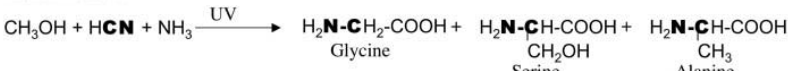

FIG. 6.-Pathway of atoms from starting materials in (a) the glycine and serine produced in our experiments and $(b)$ the predictions made using the Strecker synthesis or the radical-radical mechanism from Woon (2002). The carbon and nitrogen atoms that originate from the HCN are in bold. The molecules produced in our experiments $(a)$ do not match the predictions made in part $(b)$.

$\mathrm{NH}_{3}$. The greatly reduced yield of serine in the absence of $\mathrm{NH}_{3}$ suggests that $\mathrm{NH}_{3}$ may play a catalytic role in serine's formation, even though there does not appear to be a direct contribution of the nitrogen from $\mathrm{NH}_{3}$ to serine. Our observation of amino acid formation in $\mathrm{HCN}$-deficient ices agrees with previous reports (Muñoz Caro et al. 2002).

We can compare our observed pathways with two specific mechanistic predictions made about these $\mathrm{H}_{2} \mathrm{O}: \mathrm{CH}_{3} \mathrm{OH}: \mathrm{HCN}$ : $\mathrm{NH}_{3}$ ices. A Strecker-type synthesis, in which the $\mathrm{CH}_{3} \mathrm{OH}$ is initially oxidized via UV photolysis to $\mathrm{HCHO}$, followed by a reaction between $\mathrm{HCHO}, \mathrm{HCN}$, and $\mathrm{NH}_{3}$ to form glycine has been suggested (Bernstein et al. 2002). In this synthesis, the $\mathrm{NH}_{3}$ would provide the nitrogen, while $\mathrm{HCN}$ would provide the acid carbon and $\mathrm{CH}_{3} \mathrm{OH}$ the central carbon (see Fig. $6 b$ ). In contrast, a radical-radical mechanism in which dehydrogenation of $\mathrm{CH}_{3} \mathrm{OH}$ and $\mathrm{H}_{2} \mathrm{O}$ combined with hydrogenation of $\mathrm{HCN}$ forms glycine was proposed in Woon (2002). In this mechanism, both the central carbon and the nitrogen in glycine would arise from $\mathrm{HCN}$, with the acid carbon from $\mathrm{CH}_{3} \mathrm{OH}$ (see Fig. $6 b$ ). The radicalradical mechanism also predicts the formation of serine and alanine in these ices, which the Strecker synthesis fails to explain.

Neither of the suggested mechanisms adequately explains our observations. The Strecker-type synthesis may be a minor contributor to the formation of glycine, as we observed some glycine formed which matched the expected Strecker-type distribution. This may arise during acid hydrolysis, as glycine monomer is seen in the unhydrolyzed residue (Bernstein et al. 2002). No unhydrolyzed experiments were performed in the current study. However, the Strecker-type synthesis does not explain the formation of serine and alanine, nor does it match the major product pathway. In addition, the Strecker-type synthesis cannot occur in the absence of either $\mathrm{HCN}$ or $\mathrm{NH}_{3}$, meaning that it cannot be responsible for the amino acid synthesis observed in the ices lacking one of those components. Again, our results correlate well with previous reports. For example, Muñoz Caro et al. (2002) observed the formation of 16 amino acids in their photolyzed $\mathrm{H}_{2} \mathrm{O}: \mathrm{CH}_{3} \mathrm{OH}: \mathrm{NH}_{3}: \mathrm{CO}: \mathrm{CO}_{2}$ ice mixtures. The lack of $\mathrm{HCN}$ in their mixture rules out a Strecker-type synthesis. In addition, they observed production of $\beta$-alanine and diamino acids, which cannot be formed by the Strecker synthesis alone but may be the result of a radical-radical mechanism.
The Woon (2002) radical-radical mechanism accurately predicts that the nitrogen and central carbon in both serine and glycine derive from $\mathrm{HCN}$, but fails in predicting the origin of the acidic carbon. This mechanism, however, assumes that the amino acids are fully formed by the ice photochemistry. In fact, the amino acids are only observed on hydrolysis of the ice residues, suggesting that amino acid precursors are formed by the photochemistry and that these precursors are then converted to amino acids by hydrolysis. Such precursors could include nitriles, in which the acid group of the amino acid is replaced by a $-\mathrm{C} \equiv \mathrm{N}$ group; this is a reasonable assumption, as nitriles have been shown to be more photostable than amino acids, giving them a better survivability in the interstellar medium (Bernstein et al. 2004). If the radical-radical mechanism suggested by Woon were modified to encompass formation via nitriles rather than acids, it might attribute this nitrile to a starting $\mathrm{HCN}$ molecule. This nitrile carbon would then become the acid carbon in the amino acids, and the resulting pathway would match our results. Although this mechanism appears possible, the calculations necessary to determine the likelihood of this nitrile pathway have not yet been performed.

\subsection{Astrophysical and Meteoritic Implications}

The fact that our observations do not match the specific predictions made by the Strecker-type synthesis or the proposed radical-radical mechanism reveals the complexity of this type of chemistry and the need for more theoretical studies of these reactions. Our observations suggest several pathways, which in turn suggest that the formation of amino acids in interstellar ices may be a somewhat robust reaction, not tied to a specific ice composition. This observation is in agreement with previous laboratory experiments in which amino acids have been made from several different ice compositions (Bernstein et al. 2002; Muñoz Caro et al. 2002; Holtom et al. 2005).

The compositions and UV dosage in this study are reasonable estimates for conditions at the edge of a protostar or even within a dense interstellar cloud. However, specific environments and compositions of ices within such clouds will, of course, vary over a range (Gibb et al. 2004). Our observations of multiple pathways for glycine formation indicate that the synthesis of glycine precursors may occur in a variety of ice compositions; for example, although HCN appears to be necessary for the major pathway, glycine may still be formed in the absence of HCN. Again, this observation points toward a somewhat robust formation of glycine in interstellar ices that is not rigidly dependent on ice composition.

If amino acids do form in a variety of presolar ices, they may contribute to the inventory of compounds detected in Murchison and other carbonaceous meteorites or found in comet samples. If so, the distribution of meteoritic amino acids may provide some insight into the ice conditions. For example, serine is a major product in our $\mathrm{H}_{2} \mathrm{O}: \mathrm{CH}_{3} \mathrm{OH}: \mathrm{HCN}: \mathrm{NH}_{3}$ ices, but is not a significant component of the Murchison amino acids (Sephton 2002). However, we note that the pathway for serine formation in our ices is strongly dependent on $\mathrm{HCN}$, more so than the pathway for the other amino acids we detected, glycine and alanine. The relative lack of serine in Murchison suggests that amino acids not formed during aqueous alteration on the parent body could have formed in an HCN-poor ice.

Unfortunately, the multitude of pathways leading to these amino acids makes it difficult to attempt to tie the isotopic ratios in the meteoritic compounds to any specific interstellar species. Although the glycine produced in our ices obtains the majority of its nitrogen and carbon from $\mathrm{HCN}$, we cannot presume that the ${ }^{15} \mathrm{~N} /{ }^{14} \mathrm{~N}$ and ${ }^{13} \mathrm{C} /{ }^{12} \mathrm{C}$ ratios in meteoritic glycine reflect the 
interstellar HCN ratios present in the ices that may have formed this compound. The possibility of minor pathways in which nitrogen arises from $\mathrm{NH}_{3}$ or carbon from $\mathrm{CH}_{3} \mathrm{OH}$ precludes this type of analysis.

It may, however, be possible to test the potential link between interstellar ice mechanisms of formation of amino acids and meteoritic compounds. For example, the major pathway we observed for glycine formation in interstellar ice analogs has both carbon atoms arising from the HCN. If this is an important pathway for meteoritic glycine, then both carbon atoms in the majority of meteoritic glycine should show similar ${ }^{13} \mathrm{C} /{ }^{12} \mathrm{C}$ ratios. Conversely, if a Strecker-type synthesis on the parent body is responsible for meteoritic glycine formation, then the ${ }^{13} \mathrm{C} /{ }^{12} \mathrm{C}$ ratios of the acid carbon and central carbon are not required to be correlated. Similarly, the carbons deriving from $\mathrm{CH}_{3} \mathrm{OH}$ would presumably carry its precursor deuterium enrichment, while carbons arising from $\mathrm{HCN}$ may contain deuterium enrichments determined by the $\mathrm{H}_{2} \mathrm{O}$ that protonates the $\mathrm{HCN}$. Thus, if isotopic ratios of individual atoms in the meteoritic amino acids can be tested, which is admittedly an analytically difficult proposition, it may be possible to distinguish between formation in ice chemistry and on parent bodies.

\section{CONCLUSIONS}

We have observed multiple pathways for the formation of the amino acids glycine, serine, and alanine in interstellar ice analogs. Although major pathways rely on the presence of $\mathrm{HCN}$ in the ices, amino acids are formed in the absence of HCN as well. The pathways we observe do not match predictions previously made for the mechanisms involved in this ice chemistry; neither a Strecker-type synthesis (Bernstein et al. 2002) nor the specific radical-radical mechanism suggested by Woon (2002) adequately explain the disposition of atoms in the product molecules. A modified radical-radical mechanism that takes into account the formation of nitriles as amino acid precursor molecules may explain the results.

The multiple pathways to amino acid formation suggest that these reactions are not narrowly dependent on ice composition, but may proceed under a variety of compositional and environmental conditions, with different product distributions observed. Amino acids produced in this manner may contribute to the inventory of meteoritic amino acids. A comparison of meteoritic distributions to amino acid distributions formed under various conditions may provide further information on the potential link between ice chemistry and meteoritic organics.

We are grateful for funding from the NASA Astrobiology Institute (both the NASA Ames team and the Goddard Center for Astrobiology), the NASA Exobiology Program, the NASA Origins of Solar Systems program, and a NAI-NASA Postdoctoral Fellowship for J. E. E. We also thank Robert Walker and Daniel Glavin for technical assistance.
Allamandola, L. J., Sandford, S. A., Tielens, A. G. G. M., \& Herbst, T. M. 1992, ApJ, 399, 134

Allamandola, L. J., Sandford, S. A., \& Valero, G. J. 1988, Icarus, 76, 225

Bernstein, M. P., Ashbourn, S. F. M., Sandford, S. A., \& Allamandola, L. J. 2004, ApJ, 601, 365

Bernstein, M. P., Dworkin, J. P., Sandford, S. A., Cooper, G. W., \& Allamandola, L. J. 2002, Nature, 416, 401

Bernstein, M. P., Sandford, S. A., Allamandola, L. J., Chang, S., \& Scharberg, M. A. 1995, ApJ, 454, 327

Blagovich, V., Petrie, S., \& Bohme, D. K. 2003, MNRAS, 339, L7

Boonman, A. M. S., et al. 2001, ApJ, 553, L63

Chakrabarti, S., \& Chakrabarti, S. K. 2000, A\&A, 354, L6

Cottin, H., Moore, M. H., \& Benilan, Y. 2003, ApJ, 590, 874

Cronin, J. R., \& Chang, S. 1993, in The Chemistry of Life's Origins, ed. J. M. Greenberg, C. X. Mendoza, \& V. Pirronelle (Dordrecht: Kluwer), 209

Cronin, J. R., Cooper, G. W., \& Pizzarello, S. 1995, Adv. Space Res., 15, 91

Cronin, J. R., \& Moore, C. B. 1971, Science, 172, 1327

Cronin, J. R., \& Pizzarello, S. 1983, Adv. Space Res., 3, 5

Ehrenfreund, P., Bernstein, M. P., Dworkin, J. P., Sandford, S. A., \& Allamandola, L. J. 2001a, ApJ, 550, L95

Ehrenfreund, P., Glavin, D. P., Botta, O., Cooper, G., \& Bada, J. L. 2001b, Proc. Natl. Acad. Sci., 98, 2138

Epstein, S., Krishnamurthy, R. V., Cronin, J. R., Pizzarello, S., \& Yuen, G. U. 1987, Nature, 326, 477

Gibb, E. L., Whittet, D. C. B., Boogert, A. C. A., \& Tielens, A. G. G. M. 2004, ApJS, 151, 35

Glavin, D. P., Dworkin, J. P., Aubrey, A., Botta, O., Doty, J. H., III, Martins, Z., \& Bada, J. L. 2006, Meteoritics Planet. Sci., 41, 889

Holtom, P. D., Bennett, C. J., Oramura, Y., Mason, N. J., \& Kaiser, R. I. 2005, ApJ, 626, 940

Kuan, Y.-J., Charnley, S. B., Huang, H.-C., Tseng, W.-L., \& Kisiel, Z. 2003, ApJ, 593, 848

Kvenvolden, K., Lawless, J., Pering, K., Peterson, E., Flores, J., Ponnamperuma, C., Kaplan, I. R., \& Moore, C. 1970, Nature, 288, 923

Lacy, J. H., Faraji, H., Sandford, S. A., \& Allamandola, L. J. 1998, ApJ, 501, L105

Largo, A., Redondo, P., \& Barrientos, C. 2003, Int. J. Quantum Chem., 98, 355

Lerner, N. R. 1997, Geochim. Cosmochim. Acta, 61, 4885

Lerner, N. R., \& Cooper, G. W. 2005, Geochim. Cosmochim. Acta, 69, 2901

\section{EFERENCES}

Loeffler, M. J., Baratta, G. A., Palumbo, M. E., Strazzulla, G., \& Baragiola, R. A. 2005, A\&A, 435, 587

MacKenzie, S. L., Tenaschuk, D., \& Fortier, G. 1987, J. Chromatography, 387, 241

Mathis, J. S., Mezger, P. G., \& Panagia, N. 1983, A\&A, 128, 212

Mawhinney, T. P., Robinett, R. S. R., Atalay, A., \& Madson, M. A. 1986, J. Chromatography, 358, 231

Mendoza, C., Ruette, F., Martorell, G., \& Rodriguez, L. S. 2004, ApJ, 601, L59

Mennella, V., Baratta, G. A., Esposito, A., Ferini, G., \& Pendleton, Y. J. 2003, ApJ, 587, 727

Muñoz Caro, G. M., et al. 2002, Nature, 416, 403

Oro, J., Gibert, J., Lichtenstein, H., Wikstrom, S., \& Flory, D. A. 1971, Nature, 230,105

Palumbo, M. E., Strazzulla, G., Pendleton, Y. J., \& Tielens, A. G. G. M. 2000, ApJ, 534, 801

Peltzer, E. T., \& Bada, J. L. 1978, Nature, 272, 443

Peltzer, E. T., Bada, J. L., Schlesinger, G., \& Miller, S. L. 1984, Adv. Space Res., 4, 69

Pizzarello, S., Feng, X., Epstein, S., \& Cronin, J. R. 1994, Geochim. Cosmochim. Acta, 58, 5579

Pizzarello, S., \& Huang, Y. 2005, Geochim. Cosmochim. Acta, 69, 599

Pizzarello, S., Krishnamurthy, R. V., Epstein, S., \& Cronin, J. R. 1991, Geochim. Cosmochim. Acta, 55, 905

Prasad, S. S., \& Tarafdar, S. P. 1983, ApJ, 267, 603

Rodier, C., Sternberg, R., Raulin, F., \& Vidal-Madjar, C. 2001, J. Chromatography A, 915, 199

Sandford, S. A., Bernstein, M. P., \& Dworkin, J. P. 2001, Meteoritics Planet. Sci., 36, 1117

Sephton, M. A. 2002, Natl. Prod. Rep., 19, 292

Snyder, L. E., \& Buhl, D. 1971, ApJ, 163, L47

Snyder, L. E., et al. 2005, ApJ, 619, 914

Sorrell, W. H. 2001, ApJ, 555, L129

Tsugita, A., Uchida, T., Mewes, H. W., \& Ataka, T. 1987, J. Biochem., 102, 1593

Warneck, P. 1962, Appl. Optics, 1, 721

Woon, D. E. 2002, ApJ, 571, L177

Wu, R., Judge, D. L., Chen, H., Lu, H., \& Cheng, B. 2005, EOS Trans., 86, abs. P11B0119

Yuen, G., Blair, N., Marais, D. J. D., \& Chang, S. 1984, Nature, 307, 252

Zhao, M., \& Bada, J. L. 1995, J. Chromatography A., 690, 55 